Bull. Mater. Sci., Vol. 19, No. 1. February 1990, pp. 3. 14. ("Printed in India.

\title{
Developments in nuclear techniques for hydrogen depth profiling
}

\author{
D K AVASTHI \\ Nuclear Science Centre, Aruna Asaf Ali Marg, Post Box 10502, New Delhi 110067, India
}

\begin{abstract}
bstract. Hydrogen plays a crucial role in the properties of various materials, devices and in growth of hydrogenated amorphous materials. Therefore. its quantification and determination of concentration at various depths is of immense interest. Among various techniques of hydrogen depth profiling, the nuclear techniques being non-destructive in nature are widely used. Elastic recoil detection analysis (ERDA) with helium ions was first used for hydrogen depth profiling. Later on, the heavy ions were utilised due to certain advantages. It has been shown recently by us that $\mathrm{H}$ alongwith other elements in thin film up to $\mathrm{Ca}$ can be detected simultaneously with heavy ion ERDA, if the elements being detected are well separated in masses. It also has been shown that ever the neighbouring elements such as $\mathrm{C}, \mathrm{N}, \mathrm{O}$ alongwith $H$ can be detected provided a $\triangle E-E$ detector telescope is used in ERDA. The time of fight (TOF) with ERDA, nuclear reaction analysis (NRA), neutron TOF are other nuclear techniques for hydrogen profiling. There are. however, certain problems that arise in nuclear techniques such as hydrogen loss during the measurement, which must be taken into account for accurate and reliable results. A scenario of developments in nuclear techniques with suitable examples is given in this brief review.
\end{abstract}

Keywords. Elastic recoil detection analysis (ERDA); $\Delta E-E$ detector telescope; time of flight (TOF); nuclear reaction analysis (NRA).

\section{Introduction}

Hydrogen being the smallest and lightest atom, diffuses easily in materials. Its presence affects various properties (e.g. electrical, mechanical, chemical and spectroscopic) of the materials. To quote a few examples : (i) it causes embrittlement in steel, aluminium alloys, etc., (ii) it affects the properties of solar cells, $\mathrm{Nb}$ based superconductors, $\mathrm{Zr}$ alloys, etc. and (iii) it plays a crucial role in the growth of diamond films, diamond like carbon (DLC) films, amorphous Si (a-Si) films etc. In some metals, it sometimes gets introduced unintentionally and causes undesirable effects. On the other hand, it is introduced intentionally for the growth of the materials like a-Si, DLC films, etc. Due to these and other possible reasons, the detection of $\mathrm{H}$ and its depth profiling is of wide interest. Indirect methods (like infrared spectroscopy, Raman spectroscopy, etc.) of measuring $\mathrm{H}$ concentration exploit the fact that hydrogen alters the physical or spectroscopic properties. These methods require a standard calibrator, which is used to quantify hydrogen. Direct methods of hydrogen profiling are time of flight with neutron scattering (Overley 1987), secondary ion mass spectroscopy (SIMS) (Lieb] 1972, 1981; Magee and Wu 1978), nuclear reaction analysis (NRA) (Liech and Tombrello 1973) and elastic recoil detection analysis (ERDA) (L'Ecuyer et al 1976; Doyle and Peercey 1979). This article mainly deals with the nuclear techniques of hydrogen profiling using the ion beams from the accelerators. The developments carried out at the Nuclear Science Centre (NSC) (Mehta and Patro 1988) in this direction are discussed in some detail and are mainly based on the elastic recoil detection analysis (ERDA). The underlying principle of the ERDA technique is given in the following section. 


\section{Principle of ERDA and mathematical formulation}

When a projectile of mass $m_{\mathrm{p}}$ strikes a target atom of mass $m_{\mathrm{t}}$ (which is at rest), scattering takes place as shown in figure 1. In the collision, the incident ion imparts some energy to the atom at rest and gets scattered at an angle $\theta$. The atom, which was at rest, then recoils with an energy $E_{\mathrm{r}}$. The energy $E_{\mathrm{r}}$ of the recoil depends on mass of the projectile $m_{\mathrm{p}}$, mass of the target $m$, energy of the projectile $E_{\mathrm{p}}$ and the recoil angle $\theta$. Mathematically, it is given by the following expression which can be derived using the laws of conservation of energy and momentum

$$
E_{\mathrm{r}}=\frac{4 m_{\mathrm{p}} m_{\mathrm{t}} E_{\mathrm{p}} \cos ^{2} \phi}{\left(m_{\mathrm{p}}+m_{\mathrm{t}}\right)^{2}} .
$$

Since projectile mass $m_{\mathrm{p}}$, its energy $E_{\mathrm{p}}$ and recoil angle remains fixed under a given experimental condition, different atoms (having different masses) present in the sample will have different recoil energies as governed by (1). Thus recoil energy spectrum can identify the masses of the atoms in the sample.

The energy of the recoils from various depths at which they originate depends on two factors: (i) rate of energy loss of incoming ion in sample material, (ii) the rate of energy loss of recoiling ion in the sample prior to emergence from the sample and (iii) the energy loss of the recoil ions in the stopper foil which is used to stop the unwanted scattered ions and recoils as shown in a schematic experimental set up in figure 2 . Using the above arguments, the depth scale can be generated in the recoil energy spectrum.

The quantitative estimate of an element in the sample can be made by measuring the number of recoils $Y$, detected for a given dose of projectiles. The number of atoms $/ \mathrm{cm}^{2}$ of an element is given by the following expression

$$
N_{\mathrm{t}}=\frac{Y \sin \alpha}{N_{\mathrm{p}}(\mathrm{d} \sigma / \mathrm{d} \Omega) \Omega},
$$

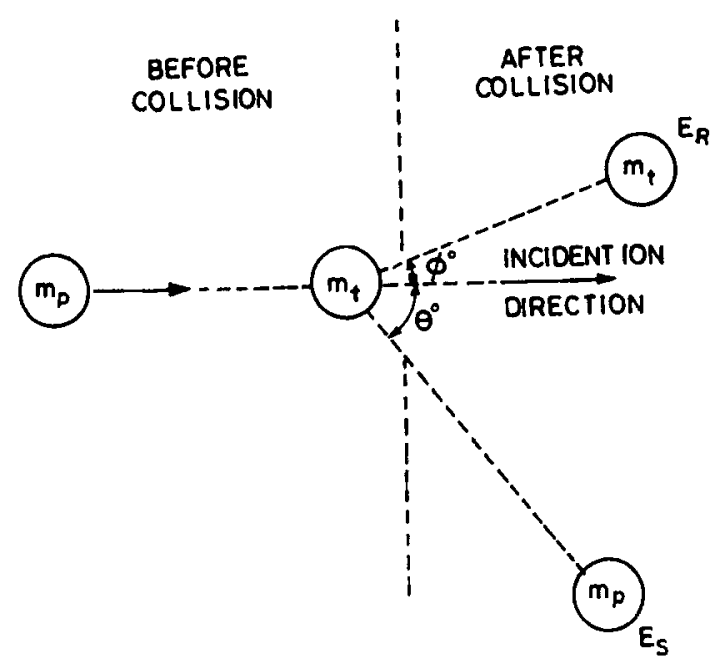

Figure 1. Schematic of ion atom collision and the symbols used in the text. 


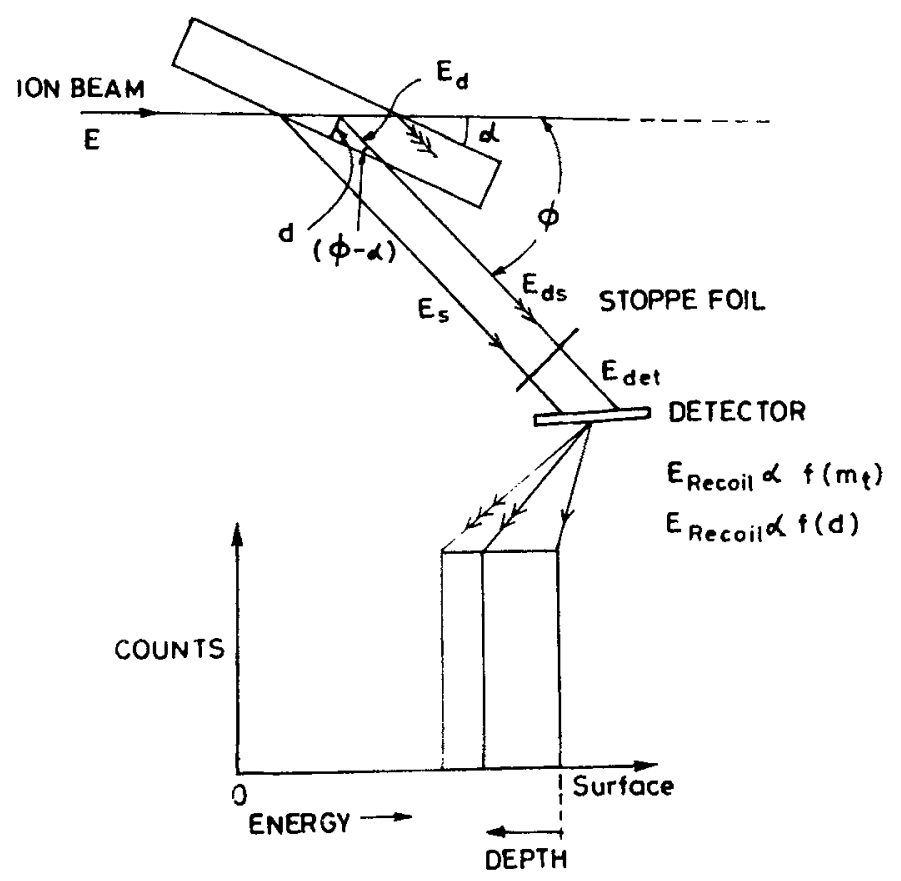

Figure 2. schematic oI ERDA set up, with the sketch to explain the principle of ERDA.

where $N_{\mathrm{p}}$ is number of projectiles incident on the sample, $\Omega$ is the solid angle subtended by the detector and $(\mathrm{d} \sigma / \mathrm{d} \Omega)_{\mathrm{r}}$ is Rutherford recoil cross section given by

$$
(\mathrm{d} \sigma / \mathrm{d} \Omega)=\left[\frac{Z_{\mathrm{p}} Z_{\mathrm{t}} e^{2}\left(m_{\mathrm{p}}+m_{\mathrm{t}}\right)}{2 E_{\mathrm{p}} m_{\mathrm{t}}}\right]^{2} \frac{1}{\cos ^{3} \bar{\phi}},
$$

where $Z_{\mathrm{p}}$ and $L_{\mathrm{t}}$ are atomic numbers of projectile and target atoms respectively. Other symbols have their usual meaning as mentioned earlier.

\section{Hydrogen depth profiling}

Energy to depth scale conversion of the recoil spectrum is carried out using the following algorithim.

The energy of recoil from the surface is given by $E_{\mathrm{s}}$ expressed as

$$
E_{\mathrm{s}}=K E
$$

where $K$ is the kinematic factor given by

$$
K=\frac{4 m_{\mathrm{p}} m_{\mathrm{t}} \cos ^{2} \phi}{\left(m_{\mathrm{p}}+m_{\mathrm{t}}\right)^{2}}
$$

From the schematic in figure 2, one can see that recoils from the surface have the maximum energy represented by single arrow. Thus maximum recoil energy represents the surface. The energy of hydrogen recoils originating from a depth $d$ is given by $E_{\mathrm{d}}$ 
and is represented by double arrows in figure 2. $E_{\mathrm{d}}$ can be explained as

$$
E_{\mathrm{d}}=K\left[E-\frac{d}{\sin \alpha} \cdot(\mathrm{d} E / \mathrm{d} x)_{\text {in }}\right],
$$

where $(\mathrm{d} E / \mathrm{d} x)_{\text {in }}$ is the stopping power of material for the incoming ion, The $\mathrm{H}$ recoil energy after coming out of the surface originating at depth $d$ is

$$
E_{\mathrm{ds}}=E_{\mathrm{d}}-\frac{d}{\sin (\phi-\alpha)} \cdot(\mathrm{d} E / \mathrm{d} x)_{\mathrm{oul}},
$$

where $(\mathrm{d} E / \mathrm{d} x)_{\text {out }}$ is the stopping power of material for $\mathrm{H}$ recoil. The $\mathrm{H}$ recoil energy as detected by the detector is given by

$$
E_{\mathrm{det}}=E_{\mathrm{ds}}-\Delta E_{\mathrm{foil}}\left(E_{\mathrm{ds}}\right)
$$

where $E_{\mathrm{foil}}\left(E_{\mathrm{ds}}\right)$ is the energy loss of the recoil ion in the stopper foil, which is function of recoil energy $E_{\mathrm{ds}}$.

Depth resolution is the quantity of interest in any measurement of depth profiling. Depth resolution here is expressed by the following relation

$$
\mathrm{d}_{\mathrm{res}}=\frac{\mathrm{d} E}{\left[\frac{K S_{\mathrm{e}}}{\sin \alpha}+\frac{S_{\mathrm{ke}} K E}{\sin (\phi-\alpha)}\right]},
$$

where $\mathrm{d} E$ is the energy resolution of the detection system which includes, detector resolution, kinematic broadening, straggling due to foil, etc. $S_{\mathrm{e}}$ and $S_{\mathrm{ke}}$ are the stopping powers of the material for incident and recoil ions respectively.

Different possibilities of hydrogen profiling by ERDA and relevant developments are discussed next.

\subsection{ERDA with helium ions}

Hydrogen depth profiling by ERDA with He ions was first demonstrated by Doyle and Peercey (1979). When He ions were incident on the sample, tilted at an angle with respect to the beam direction, recoils were recorded in a detector kept in the forward direction. The scattered $\mathrm{He}$ ions as well as recoil ions of elements other than $\mathrm{H}$ were stopped in the stopper foil. The latter's thickness was such as to stop the scattered ions fully. Contents of $\mathrm{H}$ and depth profiling are carried out using (4) to (8), Normally 1 to $4 \mathrm{MeV} \mathrm{He}$ ions are used for this kind of work. These incident energies of He ions are close to the Coulomb barrier and, therefore, the deviations from the Rutherford recoil cross section do occur due to possible nuclear interaction between the incident $\mathrm{He}$ ion and the $\mathrm{H}$ atom in the sample. Therefore, one has to use the modified recoil cross section formulae (Szilagyl et al 1989) for He ions, which takes into account the nuclear interactions. If one uses a standard hydrogen sample of known $\mathrm{H}$ concentration to calibrate the measurement, one avoids worry of the departure of recoil cross section from Rutherford recoil cross-section.

Typical depth resolution of $\sim 40 \mathrm{~nm}$ and sensitivity of 0.1 at. $\%$ is achieved by this method. 


\subsection{ERDA with heavy ions}

The main advantage of using heavy ions for hydrogen depth profiling is that it avoids the need of standard $\mathrm{H}$ sample for calibration, as the Coulomb barrier for heavy ion incident on $\mathrm{H}$ is relatively higher in laboratory frame of reference and, therefore, higher energies of heavy ions can safely be used for hydrogen depth profiling. The other advantages of high energy heavy ions are as follows: (1) the use of high energy heavy ions allows depth profiling of $\mathrm{H}$ for larger depths, (2) it may not require stopper foil to stop unwanted scattered ions as the maximum scattering angle $\theta_{\max }$ (given by $\sin ^{-1} m_{\mathrm{t}} / m_{\mathrm{p}}$ ) is quite small and the detector can be kept at an angle larger than the maximum scattering angle to avoid the elastically scattered ions. However, a stopper foil may still be required of a smaller thickness in order to stop unwanted heavier recoil ions and (3) it allows the detection of other heavier elements simultaneously. Some examples of this are given in the following section.

3.2a Simultaneous detection of hydrogen alongwith other light elements: If one carries out determination of the ratio $\left(N_{\mathrm{h}} / N_{\mathrm{c}}\right)$ of $\mathrm{H}$ to $\mathrm{C}$ atoms in a DLC film by conventional ERDA with He ions, one needs parameters like density of the film and $\mathrm{d} E / \mathrm{d} x$ of $\mathrm{He}$ and $\mathrm{H}$ in DLC film, which will introduce uncertainties in the determination of $\left(N_{\mathrm{h}} / N_{\mathrm{c}}\right)$. Moreover, the errors in target angle and detector angle read outs will get reflected in the film thickness determination which is required for $\left(N_{\mathrm{h}} / N_{\mathrm{c}}\right)$. Simultaneous detection of $\mathrm{C}$ and $\mathrm{H}$ in a thin DLC film makes the determination simpler and easier without the need of the knowledge about density, $\mathrm{d} E / \mathrm{d} x$, target and detector angle, and requirement for a standard $\mathrm{H}$ sample. The details of such an experiment are described elsewhere (Avasthi et al 1994), which are given here in brief.

A collimated ion beam of $85 \mathrm{MeV} \mathrm{Ni}$ ion was incident on a DLC film (deposited on Si substrate). A detector, with a $20 \mu$ m polypropylene foil in front of it was placed at an angle of $51^{\circ}$ with respect to the beam direction to detect $\mathrm{H}$ and $\mathrm{C}$. Recoiling Si ions from the substrate were stopped in the $20 \mu \mathrm{m}$ thick polypropylene foil. The ratio of $\left(N_{\mathrm{h}} / N_{\mathrm{c}}\right)$ was determined using the following relation, after determining the area under $\mathrm{H}$ and $\mathrm{C}$ recoils i.e. $Y_{\mathrm{h}}$ and $Y_{\mathrm{c}}$ respectively from the recoil spectrum shown in figure 3 .

$$
\frac{N_{\mathrm{h}}}{N_{\mathrm{c}}}=\frac{Y_{\mathrm{h}}}{Y_{\mathrm{c}}} \cdot \frac{(\mathrm{d} \sigma / \mathrm{d} \Omega)_{\mathrm{c}}}{(\mathrm{d} \sigma / \mathrm{d} \Omega)_{\mathrm{h}}} .
$$

If the film under study is thick $(>0.4 \mu \mathrm{m}), \mathrm{H}$ and $\mathrm{C}$ recoil energies will overlap. In such cases the $N_{\mathrm{h}} / N_{\mathrm{c}}$ can be determined in either of the following ways.

(i) Depth profiling of $\mathrm{H}$ and $\mathrm{C}$ can be carrried out up to a certain depth and the area under $\mathrm{H}$ and $\mathrm{C}$ recoils can be extracted as $Y_{\mathrm{h}}$ and $Y_{\mathrm{c}}$ to use equation (10) for finding $N_{\mathrm{h}} / N_{\mathrm{c}}$. Care has to be taken to subtract the contribution of carbon recoils while taking the area under $\mathrm{H}$ recoils.

(ii) The leading edge heights $E_{\mathrm{h}}, E_{\mathrm{c}}$ of both the $\mathrm{H}$ and $\mathrm{C}$ recoils respectively can be extracted from the recoil spectrum to determine $N_{\mathrm{h}} / N_{\mathrm{c}}$ at the surface. Here, too, the contribution of $C$ recoils should be subtracted while determining edge height $E_{\mathrm{h}}$ of $\mathrm{H}$ recoils.

Another example of simultaneous detection of light elements alongwith $\mathrm{H}$ is provided by the analysis of a-SiN $\mathrm{S}_{x}: \mathrm{H}$ film on SS backing using ${ }^{58} \mathrm{Ni}$ ions, the details of 


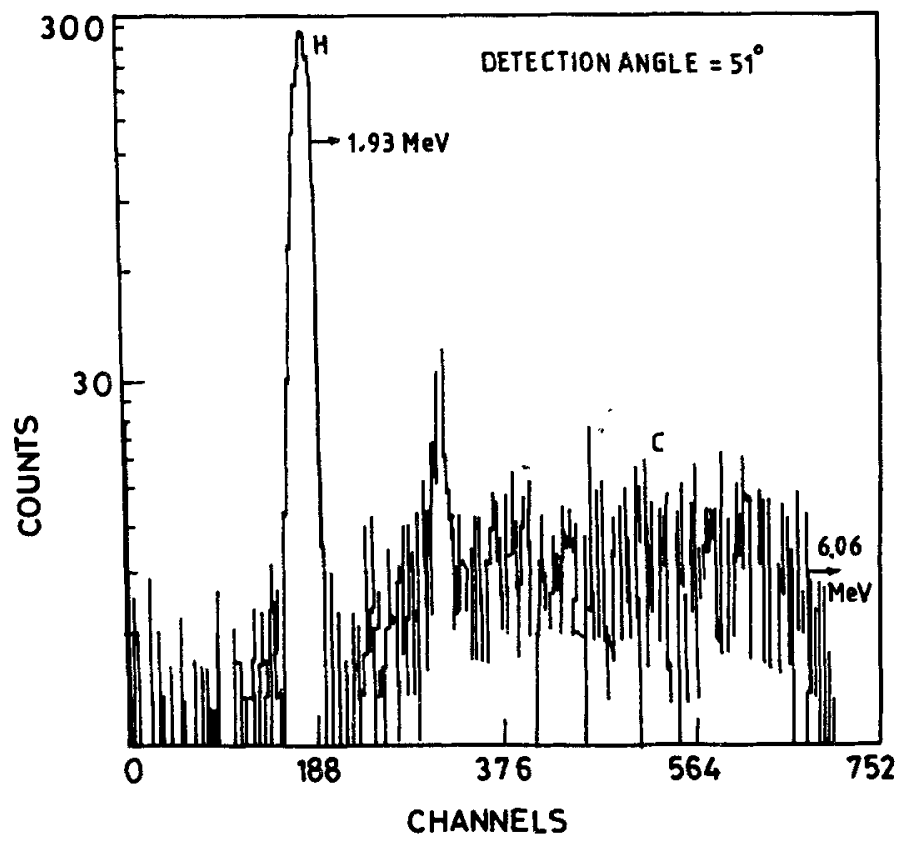

Figure 3. Recoil spectrum indicating $\mathrm{H}$ and $\mathrm{C}$ recoils of a DLC film, when $85 \mathrm{MeV}^{58} \mathrm{Ni}$ ions were used.

which are described by us (Avasthi et al 1994) elsewhere. In this case, H, N and Si recoils were seen simultaneously as shown in figure 4 . $\mathrm{H}$ depth profile in the same material is shown in figure 5 . The depth scale is shown on the top of the recoil spectrum.

The analysis of thin self supporting film (within $500 \mathrm{~nm}$ ) becomes very simple using ERDA technique. One such example (Kabirajet al 1994) is the analysis of hygroscopic and highly oxidising $\mathrm{Ca}$ self supporting film on thin $\mathrm{C}$ foil backing. Schematic of experimental set-up (ERDA in transmission geometry) and the recoil spectrum are shown in figure 6. It clearly indicates that $\mathrm{H}$ alongwith the light elements (up to $\mathrm{Ca}$ ) in a thin film can be detected easily using heavy ion ERDA technique provided the masses of the elements in the film are well separated.

3.2b ERDA with $\triangle E-E$ detector telescope: $\mathrm{H}$ alongwith other elements can be detected as long as the masses of the elements to be detected are well separated. However, if the neighbouring elements such as $\mathrm{C}, \mathrm{N}, \mathrm{O}$ etc. are to be detected with $\mathrm{H}$, one needs to use a $\triangle E-E$ detector telescope to provide the required mass discrimination.

A $\Delta E-E$ detector telescope (Bromley 1985) consists of two detectors. The recoil ion loses part of its energy $\Delta E$ in first (transmission type) detector and rest of the energy $E$ is deposited in the second detector which stops the recoil ion completely. A schematic of the detector telescope alongwith the required electronics is shown in figure 7 . Total energy of the recoil is given by $E_{\text {total }}=\Delta E+E$, which is required for generating depth scale. The signal from the transmission type detector, $\Delta E$ is proportional to $M Z^{2}$ where $M$ is the mass and $Z$ is atomic number of the recoil. The quantity $M Z^{2}$ enables one to separate out different atomic sepecies even in a complex recoil energy spectrum. Stoquert et al (1989) first demonstrated the capability of detector telescope in ERDA. $\mathrm{H}$ alongwith other light elements C, N, O were reported (Avasthi et al 1994) to be 


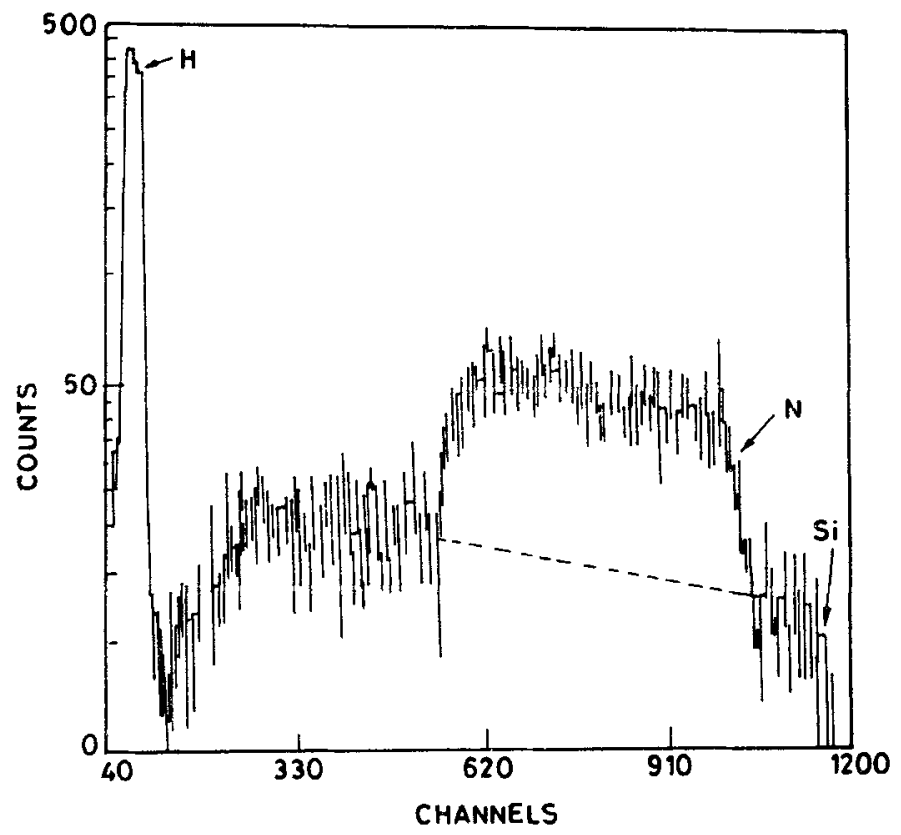

Figure 4. Recoil spectrum of an $\mathrm{a}-\mathrm{SiN}_{x}: \mathrm{H}$ film observed when $90 \mathrm{MeV}$ Ni ions were used.

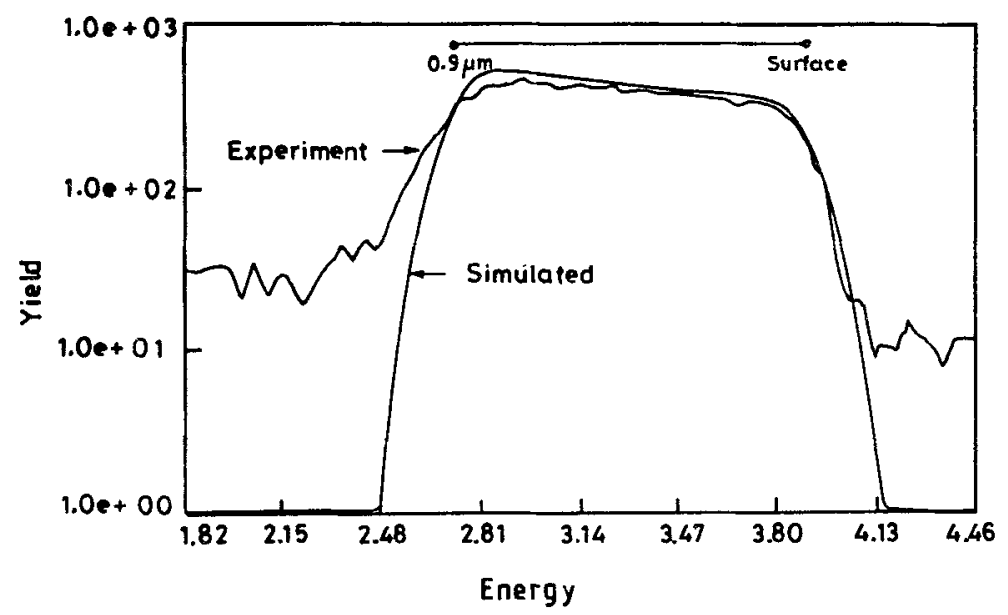

Figure 5. $\mathrm{H}$ depth profile of $\mathrm{a}-\mathrm{SiN}_{x}: \mathrm{H}$ film as determined by ERDA experiment.

detected with a sensitivity of $\sim 0.005$ at $\%$ using a $\Delta E$ gaseous and $E$ semiconductor detector. Figure 8 shows a typical two dimensional $\Delta E-E$ spectrum for the case of $\mathrm{B}$ implanted in SS. It can be seen that different bands indicating $\mathrm{H}, \mathrm{B}$ and $\mathrm{C}$ are well separated.

3.2c ERDA with time of flight (TOF): ERDA with TOF is another method which allows simultaneous detection of $\mathrm{H}$ alongwith other elements. TOF basically involves measuring the time taken by the various recoil ions to traverse a fixed distance between 


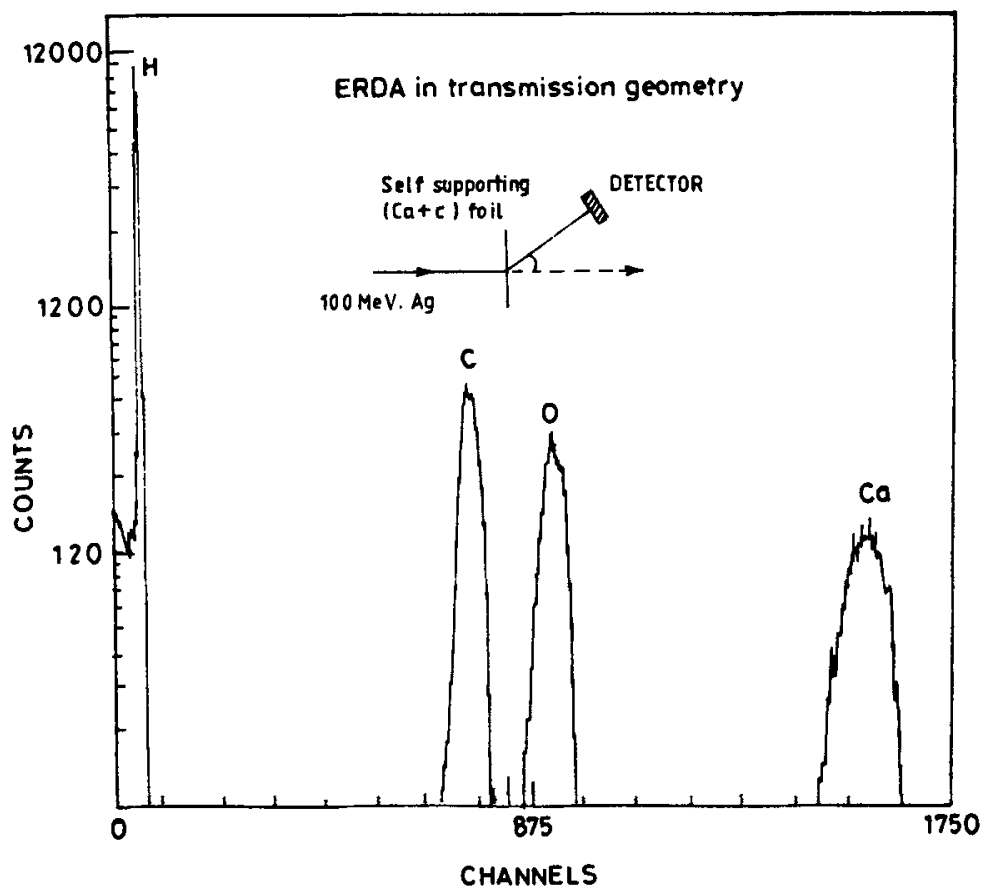

Figure 6. Schematic of ERDA in transmission geometry and the recoil spectrum of self supporting $\mathrm{Ca}$ target on thin $(\sim 50 \mathrm{~nm})$ carbon foil backing. $100 \mathrm{MeV} \mathrm{Ag}$ ions were used for this study.

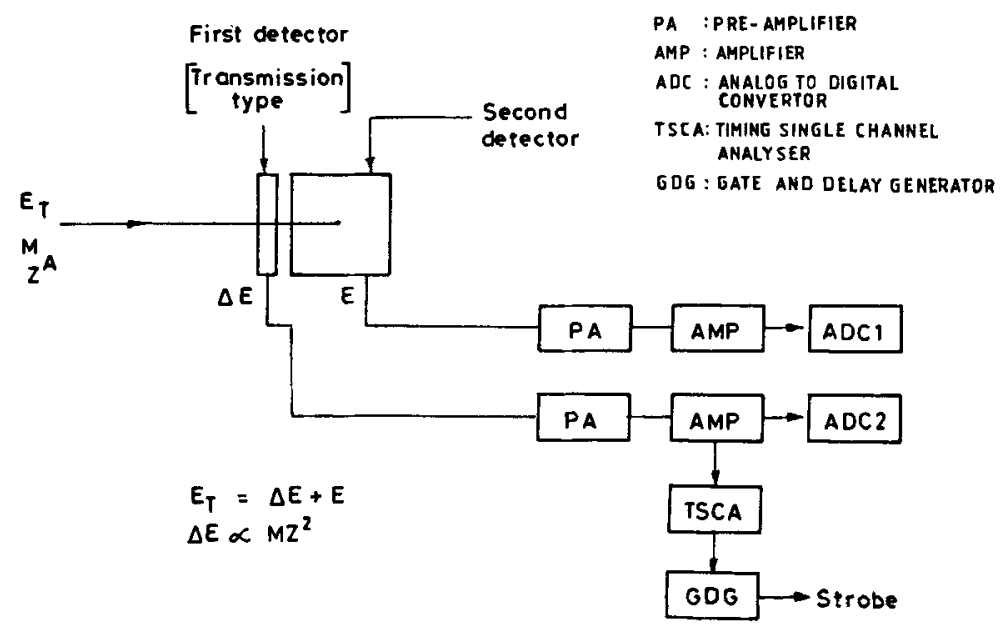

Figure 7. A schematic of detector telescope alongwith the required electronics. The signals $E$ and $\triangle E$ are stored in analog to digital converters ADC1 and ADC2, whenever there is a strobe event. The strobe event is generated by the signal in $E$ detector. Whenever an ion gives rise to a signal in $E$ detector, it also generates the signal in $\Delta E$ detector.

the two detectors. Since different recoils take different time, it allows separation of recoil of different masses. A TOF set-up requires fast timing ( $\sim$ ns rise time $)$ electronics and fast timing response detectors. Normally channeltron, microchannel plates (MCP) 


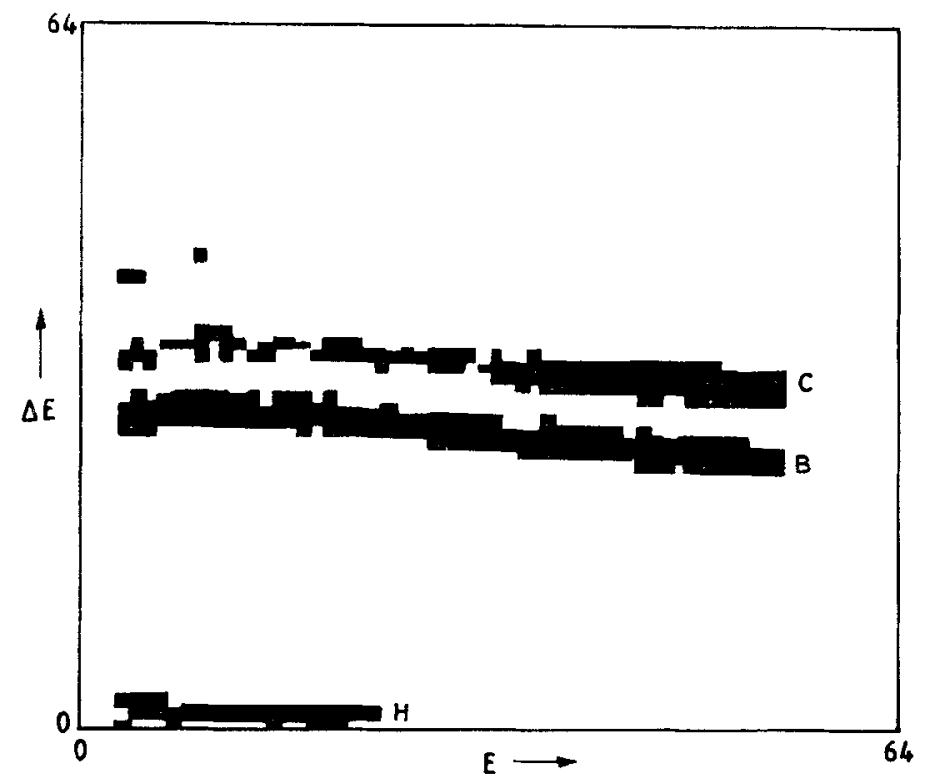

Figure 8. A two dimensional $(\Delta E-E)$ spectrum of the recoils observed at $35^{\circ}$ when $100 \mathrm{MeV}$ Ni was bombarded on B implanted SS sample.

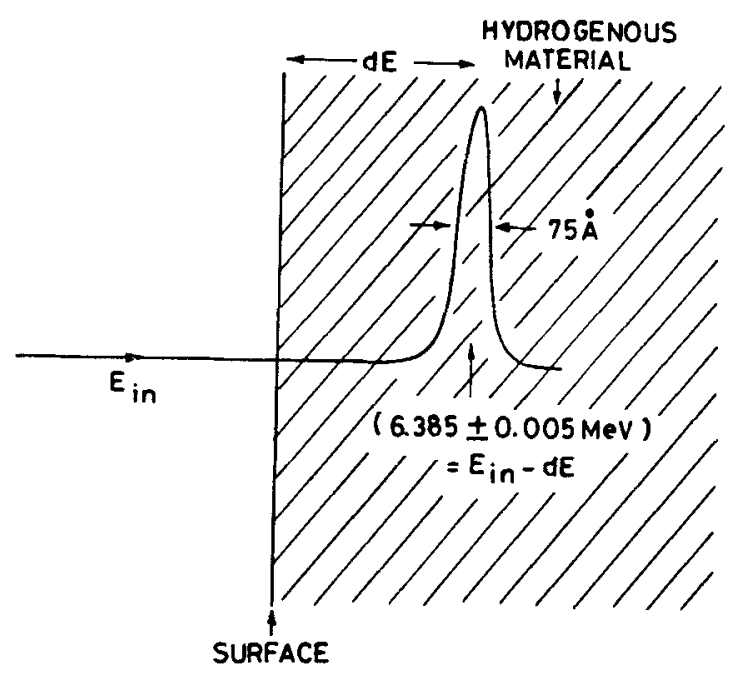

Figure 9. Schematic explaining the principle of NRA.

or overbiased fully depleted silicon surface barrier detectors are used for this purpose. In any TOF set-up, either a channeltron or MCP is used which make use of a thin carbon foil as electron emitter. The basic problem of detecting $H$ by TOF is that the efficiency of carbon foil for emitting electron in case of $\mathrm{H}$ is poor (Groleau et al 1983). It is due to the fact that all the $\mathrm{H}$ recoils which strike the carbon foil do not cause secondary electron emission (Clere et al 1973). Therefore, one requires a standard $\mathrm{H}$ sample to calibrate the TOF set-up for $\mathrm{H}$ concentration. This problem is not seen for 
Table 1. Comparison of different techniques of hydrogen depth profiling.

\begin{tabular}{|c|c|c|c|c|c|c|}
\hline $\begin{array}{l}\text { Techniques } \\
\text { for } \mathrm{H} \text { depth }\end{array}$ & Destructive & $\begin{array}{c}\text { Need of reference } \\
\text { standard }\end{array}$ & $\begin{array}{l}\text { Depth* } \\
\text { resolution }\end{array}$ & Sensitivity & $\begin{array}{l}\text { Probing } \\
\text { depth }\end{array}$ & $\begin{array}{c}\text { Multi-element } \\
\text { detection }\end{array}$ \\
\hline NRA & No & No & $5-10 \mathrm{~nm}$ & $0-1$ at. $\%$ & $<3 \mu \mathrm{m}$ & No \\
\hline ERDA with $\mathrm{He}$ & No & Yes & $30-40 \mathrm{~nm}$ & 0.1 at. $\%$ & $\sim 1 \mu \mathrm{m}$ & No \\
\hline ERDA with H.I. & No & No & $30-40 \mathrm{~nm}$ & $<0.01$ at $\%$ & $5-10 \mu \mathrm{m}$ & Yes \\
\hline $\begin{array}{l}\text { ERDA with } \Delta E-E \\
\text { telescope }\end{array}$ & No & No & $50 \mathrm{~nm}$ & 0.005 at. $\%$ & $5-10 \mu \mathrm{m}$ & Yes \\
\hline ERDA with TOF & No & Yes & $20 \mathrm{~nm}$ & $\sim 0.1$ at. $\%$ & $<5 \mu \mathrm{m}$ & Yes \\
\hline $\begin{array}{l}\text { Electron probe mass } \\
\text { analysis (EPMA) }\end{array}$ & No & No & - & 1 at. $\%$ & - & Yes \\
\hline SIMS & Yes & Yes & $-3 \mathrm{~nm}$ & 0.01 at. $\%$ & $\sim 1 \mu \mathrm{m}$ & Yes \\
\hline
\end{tabular}

* at the surface

heavier recoil ions. Thus TOF set-up is not ideally suited for $\mathrm{H}$ detection. There is, however, an advantage (Rijken 1992) of TOF technique that it improves the achievable depth resolution.

\subsection{Nuclear reaction analysis (NRA)}

The NRA technique (Liech and Tombrello 1973; Lanford et al 1976) is based on the resonant nuclear reaction between incident ${ }^{15} \mathrm{~N}$ ion and $\mathrm{H}$ in the sample. The reaction ${ }^{1} \mathrm{H}\left({ }^{15} \mathrm{~N}, \alpha \gamma\right){ }^{12} \mathrm{C}$, yields an alpha particle and a characteristic $4.43 \mathrm{MeV} \gamma$-ray. The reaction occurs at $402 \mathrm{keV}$ in centre of mass frame (equivalent to $6.385 \mathrm{MeV}{ }^{15} \mathrm{~N}$ ion energy in the laboratory frame). The width of the resonance of the reaction is $0.9 \mathrm{keV}$ and the peak cross section is 200 mbarn. The cross section off the resonance is more than 2 orders of magnitude smaller. The sample which is to be probed for $\mathrm{H}$ is bombarded with $6 \cdot 385 \mathrm{MeV}{ }^{15} \mathrm{~N}$ ions from an accelerator and $4.43 \mathrm{MeV} \gamma$-rays are detected using a $\mathrm{NaI}$ scintillation counter. If $\mathrm{H}$ is at the surface, the yield of $4.43 \mathrm{MeV}$ $\gamma$-ray is proportional to the amount of $\mathrm{H}$ at the surface. The incident ion energy is increased above $6.385 \mathrm{MeV}$ in order to determine $\mathrm{H}$ within the sample surface. As the ion traverses the sample its energy decreases and reaches the fixed resonant energy only at a certain depth inside the surface, where it produces $4.43 \mathrm{MeV} \gamma$-ray. The difference of incident ion energy and resonant energy alongwith stopping power data provides the depth scale as explained in figure 9. This typical reaction can provide an $\mathrm{H}$ analysing depth of $\sim 3 \mu \mathrm{m}$. The other popular reaction used (Liech and Tombrello 1973) is ${ }^{1} \mathrm{H}\left({ }^{19} \mathrm{~F}, \alpha \gamma\right){ }^{16} \mathrm{O}$, which gives an analysing depth of less than $1 \mu \mathrm{m}$. This technique does provide excellent depth resolution $(50-100 \AA)$ and sensitivity better than $0 \cdot 1$ at. $\%$.

\section{H loss under ion irradiation}

Certain hydrogenous materials loose hydrogen (Thomas et al 1981; Guzman et al 1985; Fujimoto et al 1988) under ion irradiation. Due care has therefore to be taken while carrying out the $\mathrm{H}$ determination by ERDA. In such cases, the contents of $H$ should be measured as a function of ion dose and the initial $\mathrm{H}$ content can be obtained by extrapolation to the zero dose condition. To minimize this problem, one can choose 
a lower mass projectile so that the incident projectiles do not impart large electronic energy excitations in the sample. It has been known from available studies (Baumann et al 1987) that $\mathrm{H}$ loss is directly related to the amount of electronic energy $(\mathrm{d} E / \mathrm{d} x)$ deposited by the projectile ion as a result of electronic excitation of the sample atoms following inelastic collisions.

$\mathrm{H}$ loss under ion irradiation, however, could be utilised to tailor the $\mathrm{H}$ concentration and $\mathrm{H}$ profile in the materials to achieve the desired properties. In fact, modification of materials by ion beam is a wide subject of study by itself, not of direct concern here.

\section{Comparison of $\mathbf{H}$ profiling techniques}

Different methods of $\mathrm{H}$ profiling have their pros and cons. Various parameters of interest such as destructive or non-destructive nature, probing depth, sensitivity, depth resolution and the capability of simultaneous multi-element detection are considered, to compare different techniques as summarized in table 1. It is worthwhile to compare the nuclear techniques with secondary ion mass spectroscopy (SIMS), which is a very popular technique for depth profiling. SIMS has better depth resolution than any other technique but it is a destructive technique. There is a peculiar problem associated with SIMS specifically for $\mathrm{H}$ measurement (Feldman and Mayer 1986). It was shown that determination of $\mathrm{H}$ quantity was dependent on partial pressure of $\mathrm{H}_{2} \mathrm{O}$ in the apparatus. It clearly indicated the need of a standard $\mathrm{H}$ sample as reference. However the choice of reference sample should be made in such a way that it has $\mathrm{H}$ affinity similar to that of the sample to be analysed. The sensitivity for $\mathrm{H}$ is also dependent on ultimate vacuum in the SIMS apparatus. The sensitivity of 0.1 at. \% is achieved only by ultra high vacuum $\left(10^{-9}\right.$ torr) UHV SIMS. The possibility of redistribution of $\mathrm{H}$ atoms in the sample during the process of analysis in SIMS cannot be ruled out. It might occur in the case of ion beam techniques too but it can be monitored in this case and therefore results are more reliable. The weaknesses of SIMS, are discussed by Mayer and Turos (1973) and Magee (1981) in detail.

\section{Conclusion}

Hydrogen detection and its depth profiling is of wide interest. Various nuclear techniques of hydrogen depth profiling, the underlying principle and the developments carried out in this field at NSC are outlined. NRA appears to be the best technique with ultimate depth resolution $(\sim 5 \mathrm{~nm})$, reasonable sensitivity $(0 \cdot 1 \mathrm{at} \%)$ and probing depth $(\sim 3 \mu \mathrm{m})$. However, it does not provide simultaneous multi-element detection. High energy heavy ions alongwith the use of $\Delta E-E$ telescope provides (i) the simultaneous multi-element detection possibility with excellent mass resolution, (ii) better sensitivity $(0.005 \mathrm{at} \%)$ and (iii) larger probing depth $(\sim 5 \mu \mathrm{m}$ for $\mathrm{H})$. However, the depth resolution $(\sim 40 \mathrm{~nm})$ is of an order of magnitude poorer as compared to NRA.

\section{Acknowledgements}

The discussions with Prof. G K Mehta had been quite fruitful in development of nuclear techniques for $\mathrm{H}$ depth profiling. The help rendered by $\mathrm{Mr} \mathrm{D}$ Kabiraj and 
Mr E T Subramniyam in the developments of technique and computer code for analysis respectively is gratefully acknowledged. The development of nuclear techniques for materials analysis at NSC was part of the project financed by the Department of Science and Technology, New Delhi.

\section{References}

Avasthi D K, Acharya M G, Tarey R D, Malhotra L K and Mehta G K 1995 Vacuum 46225

Avasthi D K, Kabiraj D, Bhagwat A, Mehta G K, Vankar V D and Ogale S B 1994 Nucl. Instrum.\& Meth. B93 480

Avasthi D K, Kabiraj D, Jaipal, Mehta G K, Barshilia H C, Somna Sah, Mehta B R and Vankar V D 1995 Vacuum 46633

Baumann H, Rupp T, Bethge K, Koild P and Wild C 1987 E-MRS Meeting, Vol. xvii p. 343

Bromley D A 1985 Treatise on heavy ion science (New York: Plenum Press) 7

Clere H G, Gerhardt H J, Richter L and Schmidt K H 1973 Nucl. Instrum. \& Meth. 113325

Doyle B L and Peercey P S 1979 Appl. Phys. Lett. 34812

L'Ecuyer J, Brassard C, Cardinal C and Terrault B 1978 Nucl. Instrum. \& Meth. 149271

Feldman L C and Mayer J W 1986 Fundamentals of surface and thin films analysis (New York: Elsevier Science Publication Co. Inc) p. 85

Fuzimoto F, Tanaka M, Iwata Y, Ootuka A, Haba M and Kobayashi K 1988 Nucl. Instrum. \& Meth. B33792

Groleau R, Gujrathi S C and Martin J P 1983 Nucl. Instrum. \& Meth. 21811

Guzman A M, Carlson J D, Bares J E and Pronko P P 1985 Nucl. Instrum. \& Meth. B7/8 468

Kabiraj D, Mandal S and Avasthi D K 1994 INTDS Conference, Bloomington, USA; 1995 Nucl. Instrum. \& Meth. A362 205

Lanford W A, Trantvetter H P, Ziegler J F and Keller J 1976 Appl. Phys. Lett. 28566

Liebl H 1972 Vacuum 22459

Liebl H 1981 Nucl. Instrum. \& Meth. 187143

Liech D A and Tombrello T A 1973 Nucl. Instrum. \& Meth. 10867

Magee C W 1981 Nucl. Instrum.\& Meth. 191297

Magee C W and Wu C P 1978 Nucl. Instrum. \& Meth. 149529

Mayer J W and Turos A 1973 Thin Solid Films 191

Mehta G K and Patro A P 1988 Nucl. Instrum.\& Meth. A268 334

Overley J C 1987 Nucl. Instrum. \& Meth. B24/25 1058

Rijken H A 1992 Nucl. Instrum. \& Meth. B64 395

Stoquert J P, Guillaume G, Hage-Ali M, Grob J J, Ganter C and Siffert P 1989 Nucl. Instrum. \& Meth. B44 184

Szilagyi E, Paszti F, Maneeaba A, Hajdu C and Kotai E 1989 Nucl. Instrum. \& Meth. B43 502

Thomas J P, Fallavier M and Tousset J 1981 Nucl. Instrcum.\& Meth. 187573 